\title{
LA POLONIA DI OGGI E LA SHOAH: LA LEGGE E LA STORIA
}

\author{
EMILIANO VITTI (*)
}

\author{
Nota presentata dal m.e. Fausto Pocar \\ (Adunanza del 14 marzo 2019)
}

SunTO. - Le relazioni dell'anno scorso tra le leadership polacca e israeliana, con la controversia riguardo le influenze (o forse l'interferenza) del governo di Varsavia sul trattamento della memoria storica dell'Olocausto e le sue implicazioni internazionali, ci portano a fare due importanti considerazioni. In primo luogo, le possibili conseguenze che la cosiddetta "Legge sull'Olocausto" può portare alla ricerca storica; inoltre, una riflessione sugli effetti negativi che un uso improprio della legge può causare a livello sociale e amministrativo, cercando di confrontare i casi della Polonia odierna e del Governatorato generale, ossia la sezione dello stato polacco occupata dai nazisti e non annessa, ma trasformata in un'entità territoriale molto particolare.

$$
* * *
$$

ABSTRACT. - Last year's relations between the Polish and Israeli leaderships, with the controversy over the influences (or perhaps interference) of the Warsaw government on the treatment of the historical memory of the Holocaust and its international implications, lead us to make two important considerations. Firstly, the possible consequences that the so-called "Holocaust law" can bring to historical research; Furthermore, a reflection on the negative effects that an improper use of the law can cause on the social and administrative level, trying to compare the cases of today's Poland and of the General Government, that is the section of the Polish state occupied by the Nazis not annexed, but transformed into a very peculiar territorial entity.

(*) Dottorando di Ricerca in Storia, Università degli Studi di Pavia, Italy.

E-mail: emiliano.vitti01@universitadipavia.it 


\section{INTRODUZIONE}

I rapporti dell'ultimo anno tra le leadership polacca e israeliana, con l'inasprisi delle polemiche sulle influenze (o forse si potrebbe dire ingerenze) del governo di Varsavia sulla trattazione della memoria storica sull'Olocausto e sui risvolti a livello internazionale, determinano, tra gli altri, due importanti spunti di riflessione. Da un lato, le possibili conseguenze che la cosiddetta "legge sull'Olocausto" può portare all'attività di ricerca storica; dall'altro, una riflessione sugli effetti negativi che un utilizzo improprio del diritto può causare sul piano sociale e amministrativo, cercando di comparare il caso dell'odierna Polonia con quello del Governatorato generale, ${ }^{1}$ ossia la porzione di stato polacco occupato dai nazisti non annesso e trasformato in una entità territoriale del tutto peculiare.

\section{IL QUADRO INTERNAZIONALE}

La cancellazione del vertice del gruppo di Visegrad a Gerusalemme dello scorso febbraio presenta alcuni interrogativi, sia di politica internazionale sia di natura storico-giuridica. Il governo di Varsavia ha reagito alle frasi del neo-ministro degli Esteri israeliano Yisrael Katz sulla questione delle corresponsabilità polacche nello sterminio degli ebrei: «Sono figlio di sopravvissuti all'Olocausto», ha dichiarato il ministro, «e attorno alla memoria della Shoah non sono accettabili compromessi. I polacchi hanno collaborato con i nazisti. Come ripeteva il premier Yizthak Shamir, e suo padre è stato ammazzato da polacchi: "Hanno succhiato l'antisemitismo con il latte delle madri". Non è una storia che si può addolcire».2

L'intervento di Katz, rivale di Netanyahu all'interno del Likud, mirava anche alla critica delle reazioni del Premier, definite dal ministro «troppo morbide», alle recenti proteste del Primo ministro polacco Mateusz Morawiecki su una frase pronunciata dallo stesso Netanyahu

1 In seguito GG.

2 https://www.corriere.it/esteri/18_giugno_27/legge-shoah-polonia-passoindietro-governo-via-tre-anni-carcere-8becb760-79de-11e8-b751-1a567fb9343a.shtml (04-03-2019). 
riguardante l'accusa di collaborazionismo a (tutti) «i polacchi», cui peraltro seguì la rettifica del governo di Tel Aviv in «alcuni polacchi». Inoltre l'ala più a destra del partito contesta la posizione dell'esecutivo di Netanyahu sulla parziale marcia indietro da parte del Sejm (la Camera dei deputati polacca), che nel giugno dello scorso anno approvò (con 388 voti a favore, 25 contrari e 5 astenuti) l'emendamento che rimuoveva la pena di tre anni di carcere prevista per chiunque attribuisca alla «nazione polacca» responsabilità nei crimini nazisti. Questa apparente "mossa distensiva" venne adottata per riaprire il dialogo di Varsavia con Bruxelles su alcune tematiche dirimenti, come l'immigrazione e la giustizia. Rimase però ferma la volontà del governo di Morawiecki di perseguire l'«obiettivo [del]la battaglia per la verità».3 La decisione del Premier di non partecipare al summit ne ha causato la cancellazione, trasformando gli incontri con i rappresentanti dei governi di Cechia, Slovacchia e Ungheria in incontri bilaterali.

Netanyahu si è rifiutato di criticare in pubblico le esternazioni del suo ministro, manifestando invece la propria preoccupazione «per il crescente rigurgito di antisemitismo [...] e per [lo sviluppo di] un nuovo tipo di antisemitismo. Gli sforzi maligni di demonizzare lo stato ebraico negano al popolo ebraico, e solo al popolo ebraico, il diritto all'autodeterminazione della nostra patria ancestrale, in modo semplice e molto chiaro. L'antisionismo è la nuova forma di antisemitismo». ${ }^{4}$

Sembra dunque tramontare, al momento, l'ipotesi di un riavvicinamento tra Tel Aviv e Varsavia, come lasciavano timidamente presagire gli sviluppi dello scorso anno. L'annuncio del 27 giugno 2018 alimentò infatti le speranze di un positivo sviluppo della vicenda: i Primi Ministri di Polonia e Israele rilasciarono una dichiarazione congiunta sulla cancellazione delle sezioni 55a e 55b dell'emendamento, che definiva come «contrario ai fatti» ogni riferimento pubblico alla nazione polacca o allo stato polacco come responsabili e/o corresponsabili per i «crimini nazisti commessi dal Terzo Reich tedesco», qualificando tali affermazioni e/o allusioni come reati penali, punibili fino a tre anni di carcere.

Un'analisi approfondita degli storici dello Yad Vashem mostra che le asserzioni storiche, presentate come fatti incontestabili nella

3 Ibid.

4 https://www.youtube.com/watch?v=LgdtQBcgP28 (04-03-2019), canale youtube di euronews. 
dichiarazione congiunta, contengono imprecisioni e scorrettezze, e che l'essenza del provvedimento rimane invariata anche dopo l'abrogazione delle sezioni di cui sopra, compresa la possibilità di danno reale ai ricercatori, a una ricerca senza impedimenti e alla memoria storica dell'Olocausto.

\section{L'APPROCCIO ALLO STUDIO DELLA STORIA}

«Si ergono innanzi alle vittime come un Gloucester coperto di sangue si ergeva dinnanzi al corpo del Re da lui ucciso e implorava la regina come ora essi implorano voi. "Di che non li ho uccisi io". E la regina rispose: "tu di che non sono stati uccisi, ma morti essi sono". Se voi giudici dichiarerete che questi uomini sono non colpevoli sarà come se aveste dichiarato: non c'è mai stata una guerra. Nessuno è stato ucciso. Nessun crimine è stato commesso». Queste parole, che citano il Re Lear di Shakespeare, appartengono alla sceneggiatura del film Il Processo di Norimberga e costituiscono la parte finale dell'arringa conclusiva del Procuratore Capo Robert Jackson (interpretato da Alec Baldwin), riferita a tutti gli imputati di quello che fu il primo di molti processi alla nomenclatura nazista.

Il profondo significato della parte finale del Processo di Norimberga è fondamentale, così come l'evoluzione che il concetto di "riconoscimento delle responsabilità" ha avuto nei decenni sia sul piano tecnico, rispetto agli studi condotti da molti storici, sia sul piano dell'attualità, specialmente di fronte alla complessa e confusa situazione politica che vediamo in questi ultimi anni in buona parte dell'Unione Europea.

La cosiddetta "legge sull'Olocausto" si inserisce perfettamente nel quadro dell'eterno dibattito su quale sia il "corretto approccio" alla Shoah e come porci di fronte alle speculazioni aventi sapore di revisionismo, sia esso di natura scientifica o politica. Il 3 marzo 2018, per esempio, una fondazione polacca vicina al partito di governo Diritto e Giustizia ha fatto ricorso alla nuova normativa denunciando un giornale argentino, reo di aver pubblicato un articolo sul pogròm di Jedwabne del luglio 1941, avvenuto con la partecipazione della popolazione polacca del luogo, che chiuse circa 300 ebrei in un fienile per poi appiccarvi il fuoco. ${ }^{5}$

5 Cfr. https://www.jewishvirtuallibrary.org/the-massacre-in-jedwabne (08-03- 
Prescindendo dalla polemica politica e dalle sue ripercussioni sul piano delle relazioni internazionali, il tentativo di amplificare le azioni in difesa degli ebrei e di descriverle come un fenomeno diffuso, ${ }^{6}$ minimizzando al contempo il ruolo dei polacchi nelle persecuzioni, costituisce, dal punto di vista dello storico, una forma di rifiuto di un corretto approccio metodologico allo studio di quelle vicende. Secondo gli storici dello Yad Vashem, la «formulazione [risulta] altamente problematica [poiché] contraddice le conoscenze storiche esistenti e accettate in questo campo, sostenendo invece efficacemente una narrativa che la ricerca ha da tempo smentito, ossia che il governo polacco in esilio e le sue organizzazioni militari sotterranee attive sul territorio polacco (Armja Krajowa) si siano spese attivamente (nella Polonia occupata e altrove) per contrastare lo sterminio degli ebrei polacchi». ${ }^{7}$ Tra le versioni contestate dagli studiosi israeliani, vi è quella sulla creazione di un «meccanismo di aiuto sistematico e di sostegno al popolo ebraico» e di una «vigorosa azione punitiva contro i polacchi che tradirono gli ebrei». ${ }^{8}$ Sebbene la dichiarazione congiunta riconosca che ci sono stati casi di polacchi che commisero crudeltà contro gli ebrei, si fa tuttavia riferimento a «numerosi polacchi» che rischiarono la vita per salvarli.

Secondo il quotidiano Haaretz, la documentazione esistente, utilizzata in decenni di ricerche storiche, fornisce un quadro completamente diverso: il governo polacco in esilio, con sede a Londra, e la Delegatura (l'organo rappresentativo del governo sul territorio polacco) non hanno agito costantemente in favore degli ebrei polacchi durante la guerra. Una buona parte della resistenza polacca, nei suoi vari movimenti, non solo non riuscì ad aiutare gli ebrei, ma fu anche non di rado attivamente coinvolta nella loro persecuzione.'

2019); J.T. Gross, A Response, Slavic Review, vol. 61, n. 3, 2002, pp. 483-9; Id. Neighbors: the destruction of the Jewish community in Jedwabne, Poland, Princeton University Press, 2001, pp. 90-104; A. Polonsky, J.B. Michlic (a cura di), The neighbors respond: the controversy over over the Jedwabne Massacre in Poland, Princeton University Press, 2004, p. 134.

6 Cfr. R.J. Golarz, M.J. Golarz, Sweet land of liberty, Bloomington, AuthorHouse, 2011, p. 95.

7 https://www.haaretz.com/israel-news/yad-vashem-historians-against-israelipolish-statement-on-shoah-bill-1.6245175 (01-03-2019).

8 Ibid.

9 Ibid. 
Sul "rapporto di proporzione" tra aiuti e persecuzioni, per gli storici dello Yad Vashem gli ultimi tre decenni di ricerche ci danno un quadro totalmente diverso: gli episodi di sostegno e aiuto dei polacchi agli ebrei durante l'Olocausto furono relativamente rari, mentre gli attacchi e persino gli omicidi di ebrei costituirono fenomeni diffusi. Nonostante le operazioni segrete di salvataggio dell'organizzazione denominata Żegota (il Consiglio per gli aiuti agli ebrei), ${ }^{10} \mathrm{col}$ sostegno del governo polacco in esilio, ${ }^{11}$ l'attività di questo organismo «non può essere proiettata sulla società polacca nel suo insieme e le sue azioni non possono essere attribuite ai gruppi di resistenza polacca in generale». ${ }^{12}$ Inoltre, sempre secondo lo Yad Vashem, lo studio dei documenti ci spiega che molti cittadini polacchi che tentarono di aiutare gli ebrei assediati dovettero guardarsi, oltre che dalla rappresaglia tedesca, anche dall'ostilità dei loro stessi connazionali. ${ }^{13}$

La dichiarazione dei due Capi di governo intendeva inquadrare separatamente la Shoah e le altre attività criminose che caratterizzarono la "quotidianità" nella Polonia occupata, in particolare all'interno del GG, sostenendo che durante la guerra «alcune persone, indipendentemente dalla loro origine, religione o visione del mondo, rivelarono il loro lato più oscuro». L'insinuazione contestata dallo Yad Vashem è che all'interno di questa definizione vengano implicitamente inclusi, da parte polacca, gli stessi ebrei. Tra i soggetti non tedeschi che mostrarono questo «lato oscuro» vi furono polacchi e cattolici: l'omissione di questo "dettaglio" da parte del governo di Morawiecki costituisce uno dei punti focali del provvedimento che promette di combattere coloro che «diffama[no] la nazione polacca». ${ }^{14}$

Da un punto di vista tecnico, vanno respinti i tentativi, di evidente

10 G.S. Paulsson, Secret City: The Hidden Jews of Warsaw, 1940-1945, Yale University Press, 2002, pp. 269-70

11 https://www.yadvashem.org/odot_pdf/Microsoft $\% 20$ Word $\% 20$ \%206392.pdf (02-03-2019).

12 https://www.haaretz.com/israel-news/.premium-yad-vashem-israel-polanddeclaration-is-historically-inaccurate-1.6244829 (03-03-2019). Riferimenti sull'organizzazione Żegota in https://sprawiedliwi.org.pl/en/o-sprawiedliwych/the-council-to-aidjews (03-03-2019).

13 https://www.haaretz.com/israel-news/.premium-yad-vashem-israel-polanddeclaration-is-historically-inaccurate-1.6244829 (03-03-2019).

14 Ibid. 
ispirazione politica, di giustapporre il fenomeno dell'antisemitismo al cosiddetto "anti-polonismo". Se da un lato risulta improprio ricorrere all'espressione "campi di sterminio polacchi", dall'altro definire l'uso di tali termini "anti-polonismo" è fondamentalmente anacronistico, scorretto sul piano dell'analisi storica e senza inerenza alcuna con l'antisemitismo, né riguardo le origini dottrinali e giuridiche, né in merito all'applicazione dei principi nazionalsocialisti sul territorio polacco occupato.

A mio avviso, sono due le considerazioni che possono spiegare come i polacchi si pongono di fronte alle delicate questioni che riguardano la loro storia recente e quali problematiche possa produrre in futuro l'atteggiamento chiuso e intransigente della classe dirigente polacca.

Il primo punto riguarda le azioni materialmente commesse da polacchi durante l'occupazione nazista. All'interno del Governatorato generale di Polonia, guidato dal giurista nazista Hans Frank, i tedeschi operarono servendosi di una larga parte del personale amministrativo polacco dei livelli più bassi. Questi cittadini polacchi, pur mantenendo vivo l'odio verso gli invasori, si adattarono alla vita del "nuovo" stato per riuscire ad avere uno stipendio e sfamare la propria famiglia. Questo può essere inteso come generale fenomeno di collaborazionismo collettivo? A mio avviso no, ma non può essere sottaciuto che una parte della macchina organizzativa che contribuì alla realizzazione dell'Olocausto, ossia quella che faceva riferimento alla Zivilverwaltung (amministrazione civile) del governo di Cracovia e delle sue diramazioni locali, fu composta da personale polacco che, pur senza poteri decisionali, partecipò a dare attuazione ai piani di sterminio. ${ }^{15}$

Un altro aspetto importante riguardò gli agenti della Gendarmerie: questa forza di polizia, che operava nei distretti locali (le cosiddette Kreise) del Governatorato come forza pubblica agli ordini diretti della Zivilverwaltung, partecipò ai rastrellamenti per il reclutamento della manodopera coatta da spedire nei campi di lavoro, come nel caso del campo di Ausschwitz III-Monowitz; l'esercizio della violenza, le intimidazioni e gli atti di brutalità commessi verso gli ebrei avveniva in quasi ogni spedizione. Una larga fetta di questi "emissari" dei capi-distretto nazisti locali era costituita da polacchi collaborazionisti.

15 E. Vitti, La destra in Polonia e l'Olocausto: problematiche di studio, Il Caffè Geopolitico, 9 maggio 2018, https://www.ilcaffegeopolitico.org/72094/la-destra-inpolonia-e-lolocausto-problematiche-di-studio (08-03-2019). 
La questione dirimente, ossia la proibizione nel definire "polacchi" i campi nazisti su territorio polacco, suona come una scusa. L'obiettivo è politico, ossia la legittimazione di una grande opera di deresponsabilizzazione collettiva, liberando dai vincoli di un'analisi scientifica tecnicamente corretta gli aspetti più marcati del nazionalismo polacco, già storicamente forte, e ora amplificato dalle derive politiche in atto nel paese.

E scorretto intendere che ogni polacco che lavorò per i nazisti (ossia eseguire gli ordini in stato di soggezione) volle farlo anche con $\mathrm{i}$ nazisti (condividendo quindi, anche solo parzialmente, gli obiettivi) e con l'intento di aiutare alla realizzazione dei programmi tedeschi sul territorio, ma impedire un libero dibattito scientifico sul tema non può che minare seriamente, oltre ai rapporti internazionali della Polonia, anche le possibilità di confronto tra gli studiosi; l'interesse primario deve rimanere il progresso della ricerca storica su una serie di avvenimenti così complessi e ancora tremendamente attuali, come l'amministrazione nei territori orientali e il funzionamento dell'universo concentrazionario nazionalsocialista. ${ }^{16}$

\section{LA LEGGE NELLA STORIA: DIRITTO E COMUNITÀ}

La cosiddetta "legge sull'Olocausto" e, più in generale, l'utilizzo della legge e del diritto influenzato da orientamenti di tipo ideologico, impone allo storico una prudente analisi attraverso un insidioso confronto con il passato; nel caso specifico, gli anni del GG.

Lo strumento giuridico, tanto oggi in un paese a vocazione nazionalista quanto ieri nel GG gestito dai nazisti, conferisce all'ideologia un ruolo nel condizionamento della ricerca storica e nella riorganizzazione della società. Tale subordinazione è individuabile nella dichiarazione rilasciata da Morawiecki il 19 febbraio scorso, replicando alle esternazioni del ministro Katz: «Le parole pronunciate dal ministro degli Esteri sono inaccettabili, non solo nella diplomazia, ma per me sono inaccettabili ovunque. La Polonia è la nazione che ha sofferto di più, insieme agli ebrei e ai rom, durante la seconda guerra mondiale». ${ }^{17}$

16 Ibid.

17 https://www.youtube.com/watch?v=LgdtQBcgP28 (04-03-2019), canale youtube di euronews. 
Secondo Karl Larenz, giurista tedesco tra i creatori della dottrina giuridica nazionalsocialista, «far parte dell'ordinamento giuridico non significa essere in relazione con altri soggetti, ma piuttosto avere un proprio determinato posto, una propria funzione, un proprio status». ${ }^{18}$ La posizione giuridica del singolo è «il suo luogo di attività [Betätigungsort] nella comunità», ${ }^{19}$ ossia una realtà non distinta dalla sfera privata, ma indentificabile con questa e quindi anche con le relazioni tra i suoi membri. Il singolo soggetto si "unifica" col suo popolo, estromettendo ogni altro soggetto o categoria di soggetti, se non riconducibili ad una appartenenza comune.

Morawiecki distingue l'elemento ebraico dallo stato polacco, sia nei provvedimenti di oggi sia nell'analisi delle sofferenze causate dall'occupazione nazista, escludendo "l'estraneo" dalla propria comunità, e relegandolo al "semplice" ruolo di "altra vittima" della violenza nazionalsocialista; si opera in tal modo una deresponsabilizzazione collettiva basata sulla visione dell'elemento polacco unicamente come vittima, condannando coloro che, a detta delle autorità di Varsavia, "insinuano il contrario".

La dichiarazione congiunta polacco-israeliana del giugno 2018 afferma l'intenzione di non intraprendere azioni legali nel campo della ricerca o dell'educazione in merito alla storia dell'Olocausto; tuttavia, questo non è chiaramente indicato nelle modifiche apportate al provvedimento. Appare evidente come l'intento del governo polacco sia stato quello di rivedere il testo senza modificarne l'essenza, a tutela e sostegno della visione politica nazionalista e sovranista.

Si pensa così di poter preservare la reputazione di un intero paese e del popolo polacco, il cui "buon nome" deve essere "salvaguardato" e protetto da "ingerenze estere", come fosse un blocco separato da ogni altro elemento "contaminatore"; tutto ciò presenta tratti corrispondenti alla visione larenziana di una «comunità che si dà nel diritto la propria forma di vita, della comunità di popolo», ${ }^{20}$ chiusa e ostile verso ogni confronto e mescolanza. Una comunità che rimane unita «nel diritto e nel sangue»: ${ }^{21} \ll$ Membro della comunità giuridica (Rechtsgenosse) è sol-

$18 \mathrm{M}$. La Torre, La «lotta contro il diritto soggettivo». Karl Larenz e la dottrina giuridica nazionalsocialista, Milano, Giuffrè, 1988, p. 181.

19 Ibid.

20 Ibid., p. 182.

21 Ibid. 
tanto colui il quale è membro della comunità di popolo (Volksgenosse); membro della comunità di popolo è colui il quale è di sangue tede$\mathrm{SCO} \gg .{ }^{22}$

Se il Primo ministro polacco, nel parlare degli anni dell'occupazione, separa il polacco dall'ebreo, opera una pesante distinzione, che ricorda la concezione larenziana dello straniero, che potrebbe essere ancora soggetto giuridico, ma con capacità giuridica assai limitata, una figura estranea alla comunità. Con questa visione distorta si configura una classificazione anche delle vittime dell'occupazione nazista, delle persecuzioni e dello sfruttamento, con soggetti di primo livello e soggetti aventi una natura sociale "marginale".

Gli amministratori tedeschi schernirono spesso l'intera popolazione del GG, ma con gli ebrei l'accanimento fu superiore: inquadrati come un "non essere", non vi era alcuna limitazione, neppure formale, agli attacchi perpetrabili contro di essi. Le operazioni di sterminio vennero anzi ricondotte all'interno di "parametri legali" conseguenti alle direttive generali scaturite dalla Conferenza di Wannsee del 20 gennaio 1942. Gli amministratori tedeschi non furono, in linea generale, solamente gli esecutori materiali di circolari e direttive provenienti dall'alto, ma parteciparono ampiamente alla progettazione di alcuni provvedimenti, personalizzandone talvolta gli aspetti relativi alla situazione dei singoli distretti.

Oltre all'influenza esercitata dalla semplice fattispecie dottrinale, che fu alla base della teoria dell'organizzazione del territorio, il rapporto con gli Ostjuden risentì di una errata percezione della loro incidenza nella vita sociale, in realtà assai inferiore alla loro presenza numerica. Gli ebrei polacchi rappresentavano nel GG circa il 15 percento della popolazione complessiva, ${ }^{23}$ con percentuali sensibilmente più alte nelle città, ma la loro effettiva partecipazione alla vita civile era trascurabile per più di una ragione. Innanzitutto, la Polonia era un paese in cui l'antisemitismo era affermato all'interno della società ben prima dell'influenza nazionalsocialista e l'accesso a posizioni istituzio-

22 K. Larenz, Rechtsperson und subjektives Recht, Grundfragen der neuen Rechtswissenschaft, vol. 6, 1935, pp. 225-60 (qui p. 246).

23 Il dato si riferisce al GG prima delle modifiche territoriali sopraggiunte nei mesi successivi all'attacco all'U.R.S.S., ossia dal 26 ottobre 1939 e il $1^{\circ}$ agosto 1941, giorno dell'annessione del Distrikt Galizien. 
nali da parte degli ebrei non fu mai benvisto dalla maggior parte dei partiti politici; inoltre la comunità ebraica polacca aveva un marcato carattere di chiusura verso il resto della popolazione; infine quelli polacchi erano, in larga maggioranza, ebrei poveri, ossia privi del "malevolo" potere di condizionamento della vita politica ed economica dettato dal denaro. ${ }^{24}$

Credo che la teoria giuridica nazionalsocialista larenziana arrivasse dunque ad «affermare una negazione», ossia si determinava l'estraneità dell'ebreo alla comunità di popolo; la politica polacca odierna e la cosiddetta "legge sull'Olocausto" conducono invece a «negare un'affermazione», ossia rifiutando la possibilità che un certo numero di soggetti polacchi abbia prestato la propria collaborazione ai tedeschi pretendendo di nasconderne le vicende al mondo. I governanti di Varsavia dimenticano l'appartenenza allo stato e al popolo polacco di 3 dei circa 5,6 milioni di ebrei uccisi nel complesso concentrazionario nazista presente in territorio polacco.

\section{CONCLUSIONI}

Il nazionalismo crescente nell'Europa comunitaria di oggi non è affatto, a mio avviso, riconducibile ad alcuna forma di dottrina giuridica e/o politica; l'unico tratto caratterizzante è costituito dal rifiuto e dalla esclusione delle diversità, in una visione omologatrice della società che rifiuta il confronto e le chiavi interpretative che può offrire la ricerca.

Negare la possibilità di studiare la storia e di fare ricerca storica liberamente, parlando dell'Olocausto e delle molte sfaccettature che lo caratterizzarono nei vari paesi europei interessati dalle deportazioni durante la guerra, porta alla negazione che ogni vittima fosse un soggetto privato di diritti, sottoposto alla dominazione nazista e condizionato nelle parole e nelle azioni.

Un paese che proibisce di raccontare un pezzo della propria storia non si preoccupa, come dichiarato da Morawiecki, della «ricerca della

24 Cfr. B. Musial, Deutsche Zivilverwaltung und Judenverfolgung im Generalgouvernement: eine Fallstudie zum Distrikt Lublin 1939-1944, Wiesbaden, Harrassowitz, 1999, p. 183. 
verità $\gg^{25}$ (espressione peraltro assai indigesta allo storico), ma esercita a sua volta un'opera che priva la comunità degli storici e l'intera società di quel paese di una fondamentale opportunità: quella dell'approfondimento della conoscenza, che consente di maturare l'effettiva consapevolezza di un popolo.

25 https://www.corriere.it/esteri/18_giugno_27/legge-shoah-polonia-passoindietro-governo-via-tre-anni-carcere-8becb760-79de-11e8-b751-1a567fb9343a.shtml (04-03-2019). 